\title{
Contenu de la fréquence de l'E.E.G. en fonction de l'effort physique et mental chez des malades présentant une insuffisance cérébrovasculaire
} (Communication préliminaire).

\author{
J. Troost, A. Kamp, H. A. C. Kamphuisen et W. Storm Van Leeuwen. \\ Medisch Fysisch Instituut T.N.O. Da Costakacade 45, Utrecht, Pays-Bas.
}

\section{INTRODUCTION.}

En pratique courante l'enregistrement de l'électroencéphalogramme (E.E.G.) est réalisé le malade étant étendu et les électrodes fixées au cuir chevelu reliées à l'appareil d'enregistrement au moyen d'un câble. Le malade est en général en état de repos relatif et l'enregistrement s'effectue durant des périodes de sommeil, de veille, avec les yeux ouverts, avec les yeux fermés, etc... Dans ces conditions, les relations entre l'E.E.G. et certaines activités ne peuvent être étudiées que de façon restreinte.

Il peut être important cependant dans le diagnostic d'un certain nombre de désordres cérébraux de procéder à l'examen E.E.G. au cours de périodes d'activité physique et mentale du malade. La réalisation d'un système radiotélémétrique E.E.G. (Storm van LEEUWEN et KAMP, 1969) a permis d'effectuer l'enregistrement chez des malades pouvant se déplacer librement, ce qui a conduit à la pratique d'examens cliniques E.E.G. d'orientation en fonction de certaines activités.

Une investigation de ce genre a été entreprise chez des malades présentant une insuffisance cérébrovasculaire. Les résultats de l'examen clinique et électroencéphalographique de routine sont souvent peu clairs chez ces malades.

Cette investigation était destinée à rechercher si des données d'E.E.G. plus spécifiques pouvaient être obtenues en soumettant ces malades à différents stress physiques et (ou) mentaux, également effectués sur des sujets de contrôle sains afin de pouvoir comparer les résultats respectifs obtenus chez les sujets malades et chez les sujets sains. Cette étude en est à un stade initial et le nombre de sujets de contrôle et de malades examinés est encore trop restreint pour permettre de tirer des conclusions. Les données obtenues permettent pourtant de montrer la nature des modifications de l'E.E.G. observées en cours de stress physique et mental.

\section{Matériel :}

L'investigation se divise en deux parties :

A) Premièrement, un groupe de malades (7) présentant un début d'insuffisance cérébro-vasculaire a été comparé à un groupe de contrôle de sujets sains (5).

B) L'investigation a été ensuite poursuivie et étendue à un groupe de malades souffrant d'une insuffisance cérébro-vasculaire manifeste.

Ce groupe a également été comparé à un groupe de contrôle sain, divisé cette fois en deux parties :

a) Un groupe de sujets sains âgés de 20 à 30 ans.

b) Un groupe de sujets sains âgés de 40 à 60 ans.

\section{Méthode :}

$1^{0}$ Pour le groupe A, la méthode suivante a été utilisée : 1'E.E.G. a été enregistré sur 12 canaux au moyen d'électrodes fixées au cuir chevelu avec du collodion (système 10-20). D'autres variables ont également été

Tirés à part : J. Troośt (à l'adresse ci-dessus). 
dérivées : l'électrocardiogramme (E.C.G.), la respiration et les mouvements oculaires. Les signaux biologiques ont été transmis à l'aide d'un système radiotélémétrique à 16 canaux et enregistrés sur papier et sur bande magnétique. L'enregistrement a eu lieu au cours de périodes de repos sur un divan d'examen et au cours de périodes d'efforts mentaux et physiques. Durant la période de repos et durant la période d'activité, les yeux de la personne examinée étaient fermés. La durée et l'intensité du stress ont été fixées individuellement et en cours du stress 1'E.C.G. et la fréquence des pulsations cardiaques ont été enregistrés et observés.

$2^{\circ}$ Pour le groupe $\mathbf{B}$, peudant la période de repos, deux techniques complémentaires ont été utilisées :

a) recherche, à l'aide d'une méthode ultrasonique reposant sur l'effet Doppler, de l'existence éventuelle d'une asymétrie du courant sanguin dans les artères carotides ;

b) les effets d'une compression alternative gauche et droite sur les carotides.

\section{Analyse :}

$1^{\circ}$ Pour le groupe A, le contenu spectral de 1'E.E.G. a été étudié à l'aide d'un analyseur de fréquence composé de 20 filtres à fréquences de résonance entre $2 \mathrm{~Hz}$ et $32 \mathrm{~Hz}$. L" "output » des filtres a été intégré durant des périodes de 30 secondes et transcrit sous forme d'histogramme.

$2^{\circ}$ Pour le groupe B, une impression provisoire concernant le contenu spectral de 1'E.E.G. a été obtenue durant les diverses situations du test « on-line » à l'aide d'un analyseur de fréquence analogique. A partir des données obtenues de cette façon, une analyse spectrale digitale a été appliquée ensuite sur les dérivations : C3P3, C4P4, Fz02, Fz01. Dans chaque situation du test, 3 analyses ont été effectuées durant une période de 12 secondes à 1'aide d'un programme d'analyse « Fast Fourier Transform ». Les données spec" trales des bandes de fréquence suivantes ont été étudiées : delta, thêta, alpha et bêta. Les autospectres, spectres de phases et fonctions de cohérence ont été déterminés pour ces bandes de fréquence. Les données de l'ordinateur ont été ensuite enregistrées graphiquement comme fonction des fréquences et un certain nombre de données spectrales ont été transcrites numériquement. Pour ce groupe, en outre, une analyse de fréquence des données obtenues à l'aide de l'effet Doppler a été pratiquée en vue d'une quantification ultérieure.

\section{I. - En ce qui concerne le groupe A :}

\section{RÉSULtats.}

Chez les sujets sains comme chez les malades, des différences de fréquence ont été observées dans les spectres de fréquence intégrés, déterminés en périodes de repos et en périodes de stress physique et mental. Dans la bande de fréquence alpha $(8-13 \mathrm{~Hz})$, cette différence consistait en un petit déplacement du maximum de la distribution de fréquence vers une fréquence plus haute ou plus basse. La capacité de résolution de fréquence de $1 \mathrm{~Hz}$ de l'analyseur s'est cependant révélée insuffisante pour ćtablir quantitativement la mesure de ce déplacement.

Pour donner malgré tout une idée des déplacements de fréquence, les rapports d'amplitude de 2 filtres à fréquences de résonance proches du maximum des spectres de fréquence ont été déterminés.

Une différence entre les rapports d'amplitude de filtre durant les périodes de rcpos et durant les périodes d'activité indiquait un déplacement de fréquence.

La hausse des rapports d'amplitude signifiait un ralentissement de la fréguence et la baisse de ces rapports signifiait une augmentation de la fréquence. En calculant les dérivations standard des divers résultats, nous avons cherché à savoir si les déplacements de fréquence étaient significatifs.

Le tableau I montre les rapports moyens d'amplitude de filtre et les dérivations standard de 3 sujets de contrôle normaux. Ces données ont été déterminées durant des périodes de repos et de stress mental.

Le tableau II montre des données correspondantes chez 3 sujets de contrôles normaux durant des périodes de repos et de stress physique.

Chez les 7 malades du groupe A, 2 ne montraient pas de maximum dans la bande de fréquence alpha. Il n'a pas été possible avec la méthode d'analyse décrite d'établir, chez ces deux patients, une modification dans la distribution de fréquence à la suite d'un stress physique ou mental. Un certain nombre des données obtenues chez les 5 autres patients figurent au tableau III. 
TABLEAU I.

\begin{tabular}{|c|c|c|c|c|c|c|c|}
\hline \multirow{2}{*}{ Sujet de contrôle } & \multirow{2}{*}{$\mathrm{fx} / \mathrm{fy}$} & \multicolumn{3}{|c|}{ Repos } & \multicolumn{3}{|c|}{ Stress mental } \\
\hline & & $\mathrm{q}$ & D.S. & $\mathrm{N}$ & q & D.S. & $\mathrm{N}$ \\
\hline $\begin{array}{lll}\text { N.P. } & 1 & \ldots\end{array} \ldots$ & $\begin{array}{l}\mathrm{f} 9 / \mathrm{f} 10 \\
\mathrm{f} 9 / \mathrm{f} 10 \\
\mathrm{f} 9 / \mathrm{f} 11\end{array}$ & $\begin{array}{l}0,85 \\
1,32 \\
1,02\end{array}$ & $\begin{array}{l}0,05 \\
0,09 \\
0,07\end{array}$ & $\begin{array}{l}4 \\
4 \\
4\end{array}$ & $\begin{array}{l}0,61 \\
1,10 \\
0,82\end{array}$ & $\begin{array}{l}0,10 \\
0,03 \\
0,02\end{array}$ & $\begin{array}{l}4 \\
4 \\
4\end{array}$ \\
\hline $\begin{aligned} \text { fx/fy } & : \text { rar } \\
\mathrm{q} & : \mathrm{mc} \\
\mathrm{N} & : \text { no }\end{aligned}$ & $\begin{array}{l}\text { ampl } \\
\text { du ra } \\
\text { spec }\end{array}$ & aplit & $\begin{array}{l}\text { Ient } \\
\text { filt } \\
\text { t'int }\end{array}$ & & $\begin{array}{l}x \text { et } y \text {. } \\
\text { ec.). }\end{array}$ & & \\
\hline
\end{tabular}

TABLEAU II.

\begin{tabular}{|c|c|c|c|c|c|c|c|}
\hline \multirow{2}{*}{ Sujet de contrôle } & \multirow{2}{*}{$f x / f y$} & \multicolumn{3}{|c|}{ Repos } & \multicolumn{3}{|c|}{ Stress physique } \\
\hline & & $q$ & D.S. & $\mathbf{N}$ & q & D.S. & $\mathbf{N}$ \\
\hline $\begin{array}{lll}\text { N.P. } & 2 & \ldots \ldots\end{array}$ & $\begin{array}{l}\text { f } 9 / \mathrm{f} 10 \\
\text { f } 10 / \mathrm{f} 12 \\
\text { f } 9 / \mathrm{f} 12\end{array}$ & $\begin{array}{l}1,32 \\
0,82 \\
1,12\end{array}$ & $\begin{array}{l}0,09 \\
0,13 \\
0,01\end{array}$ & $\begin{array}{l}4 \\
5 \\
5\end{array}$ & $\begin{array}{l}1,08 \\
0,83 \\
1,10\end{array}$ & $\begin{array}{l}0,12 \\
0,03 \\
0,05\end{array}$ & $\begin{array}{l}4 \\
4 \\
4\end{array}$ \\
\hline
\end{tabular}

TABleau III.

\begin{tabular}{|c|c|c|c|c|c|c|c|}
\hline \multirow{2}{*}{ Patient } & \multirow{2}{*}{$f x / f y$} & \multicolumn{3}{|c|}{ Repos } & \multicolumn{3}{|c|}{ Stress physique } \\
\hline & & $q$ & D.S. & $\mathrm{N}$ & $\mathrm{q}$ & D.S. & $\mathbf{N}$ \\
\hline Pt. 1. & f $9 / \mathrm{f} 10$ & 2,21 & 0,10 & 4 & 1,52 & 0,14 & 5 \\
\hline Pt. $2 \ldots$ & $\mathrm{f} 10 / \mathrm{f} 12$ & 1,14 & 0,11 & 9 & 1,49 & 0,10 & 13 \\
\hline Pt. 3.. & f $9 / \mathrm{f} 10$ & 1,15 & 0,08 & 3 & 0,90 & 0,11 & 8 \\
\hline Pt. $4 \ldots \ldots \ldots \ldots$ & f $9 / \mathrm{f} 11$ & 0,83 & 0,04 & 6 & 1,00 & 0,10 & 8 \\
\hline Pt. $5 \ldots \ldots \ldots \ldots$ & f $8 / \mathrm{f} 10$ & 1,07 & 0,06 & 3 & 1,40 & 0,14 & 3 \\
\hline
\end{tabular}

II. - En ce qui concerne le groupe B, les observations suivantes ont été faites à partir des données fournies par l'analyse spectrale digitale :

a) La réaction au stress mental s'est traduite chez quelques sujets du groupe de contrôle de même que chez quelques malades par une augmentation de la fréquence dans la bande alpha (fig. 1).

b) En ce qui concerne le stress physique, aucun des sujets de contrôle sains observés jusqu'à maintenant n'a réagi par un ralentissement de la fréquence dans la bande aipha ; certains d'entre eux ont réagi par une augmentation alors que chez les autres n'intervenait aucune variation. Chez les malades observés jusqu’à maintenant dans ce groupe, un ralentissement des fréquences de la bande alpha est intervenu dans 3 cas sur 4, le quatrième ne montrant pas de variation (voir aussi fig. 1 et 2). 

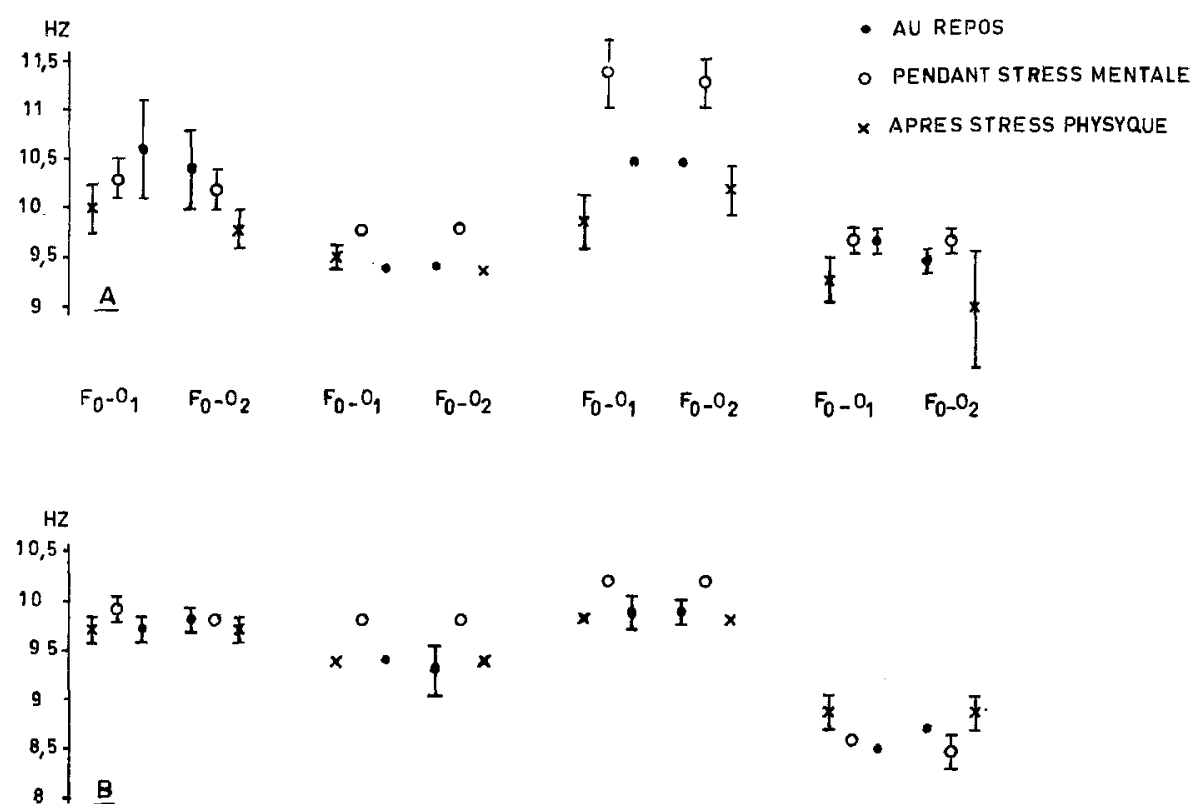

FIg. 1. - Données d'analyse spectrale digitale de 4 patients (A) et de 4 sujets de contrôle (B). Les fréquences dominantes de la bande alpha ont été déterminées au repos, durant un stress mental et après un stress physique. Chaque moyenne et chaque déviation standard ont été calculées pour trois périodes identiques de douze secondes.

\section{Discussion.}

Dans le groupe des malades présentant un début d'insuffisance cérébro-vasculaire (groupe A), certains patients ont réagi au stress physique par un ralentissement de la fréquence alpha et d'autres pas ; en outre, la technique d'analyse pratiquée pour ce groupe s'est révélée trop peu précise. C'est pourquoi nous avons poursuivi notre étude avec un groupe de malades présentant une insuffisance cérébro-vasculaire manifeste et cliniquement établie. Le groupe de contrôle a été divisé en outre en 1) un groupe de sujets sains âgés de 20 à 30 ans, dont le système vasculaire pouvait être considéré comıne parfaitement sain et 2) un groupe de sujets cliniquement sains de la même catégorie d'âge que celle des malades. A la place de l'analyse de fréquence analogique pratiquée pour le premier groupe (A) une analyse spectrale digitale, méthode considérablement plus exacte et plus sûre, a été pratiquée pour ce groupe (B).

Les résultats obtenus jusqu'à maintenant dans ce groupe ont montré que chez 3 des 4 malades présentant une insuffisance cérébro-vasculaire un ralentissement de fréquence de la bande alpha intervenait à la suite du stress physique.

Nous n'avons pas pu jusqu'à maintenant établir à l'aide de notre technique de l'effet Doppler une corrélation avec une éventuelle modification de la vitesse du courant sanguin des artères carotides. Notre but est de rechercher à l'aide de la méthode décrite, dès que nous aurons examiné suffisamment de malades, s'il existe statistiquement une éventuelle corrélation entre les données E.E.Graphiçues recueillies et les données cliniques et, si c'est le cas, l'existence d'une corrélation significative entre les résultats obtenus et l'image clinique. 

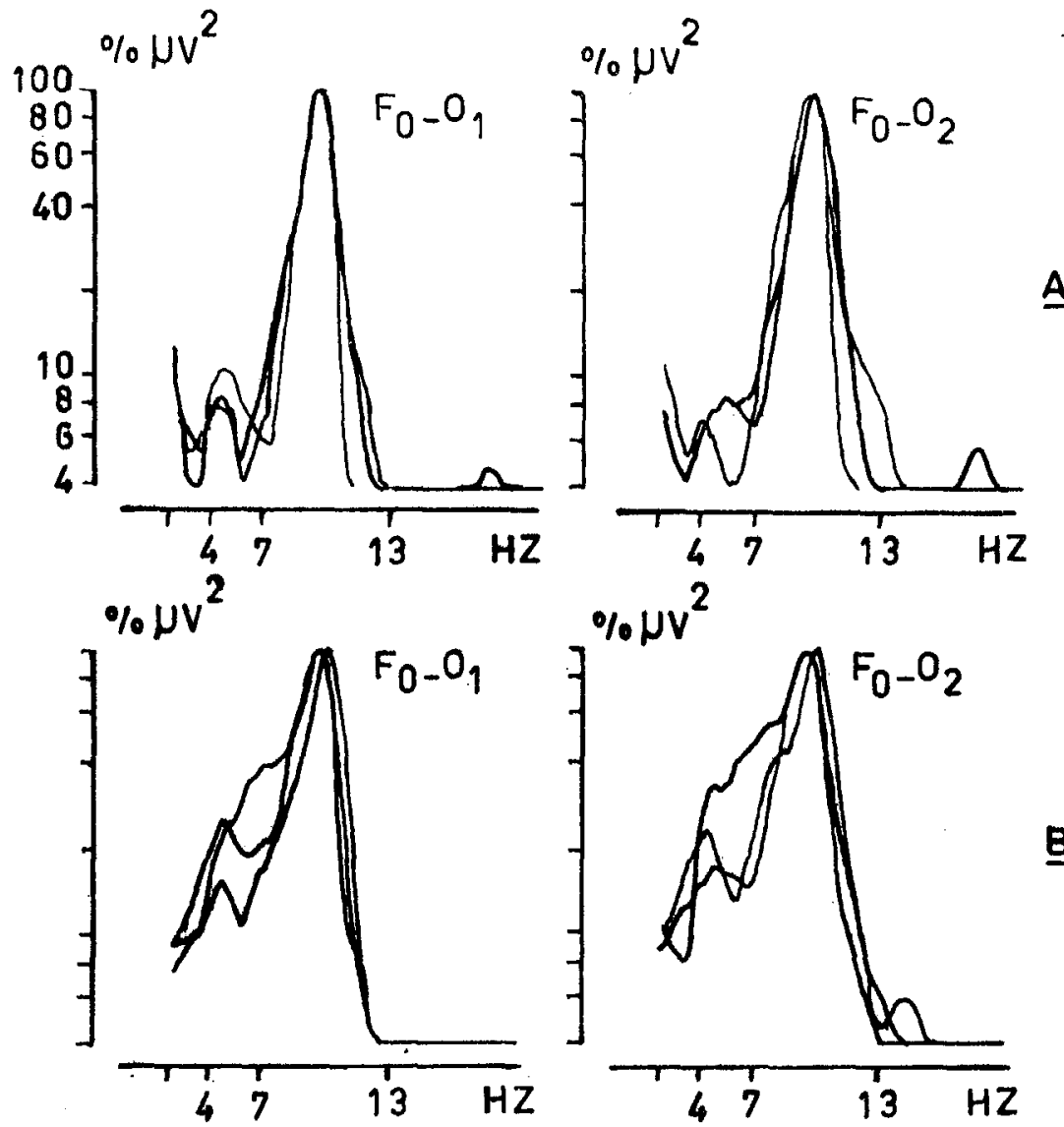

FIG. 2. - Analyses spectrales digitales des dérivations Fo-01 et Fo-02 du dernier patient (fig. 1). Superposition des 3 autospectres obtenus : $a$ ) au repos, les yeux fermés ; $b$ ) après le stress physique, les yeux fermés. Augmentation de 1'énergie dans la bande thêta après le stress physique.

\section{RéSUMÉ.}

Le contenu de la fréquence de l'E.E.G. en fonction de l'effort physique et mental chez des malades présentant une insuffisance cérébro-vasculaire (une communication préalable).

La métbode consiste en une analyse spectrale digitale de l'E.E.G., qui est enregistré à l'aide d'un système radiotélémétrique chez un groupe de malades souffrant d'une insuffisance cérébro-vasculaire. De la même façon, l'effet d'un stress physique et mental sur l'E.E.G. est étudié. Quelques données qualitatives obtenues jusqu'à maintenant avec ce procédé sont décrites.

\section{RÉFÉRENCES}

1. Grey WALTER (W.). Spontaneous oscillatory systems and alternation in stability. Neural Physiopatho$\log y, 1962$, p. 244. Edited by Robert G. Grenell, Harper and Row, New York.

2. Kalsbeek (J. W. H.). Mentale Belasting. Thèse, Amsterdam, 1967.

3. Storm van Leeuwen (W.) and Kamp (A.). Radiotelemetry of E.E.G. and other biological variables in man and dog. Proc. of the Royal Society of Medicine, May 1969, 62, 451-453. 